Research Article

\title{
Prevalence and Risk Factors Associated with Intestinal Parasitic Infection among Primary School Children in Dera District, Northwest Ethiopia
}

\author{
Dires Tegen ${ }^{1}$ and Destaw Damtie $\mathbb{B}^{2}$ \\ ${ }^{1}$ Dera Woreda Education Office, South Gondar Zone, Gondar, Ethiopia \\ ${ }^{2}$ Bahir Dar University, Department of Biology, College of Sciences, Bahir Dar, Ethiopia
}

Correspondence should be addressed to Destaw Damtie; zegades96@gmail.com

Received 15 February 2021; Revised 31 July 2021; Accepted 15 September 2021; Published 21 September 2021

Academic Editor: Julia Uhanova

Copyright (c) 2021 Dires Tegen and Destaw Damtie. This is an open access article distributed under the Creative Commons Attribution License, which permits unrestricted use, distribution, and reproduction in any medium, provided the original work is properly cited.

\begin{abstract}
Background. Globally, over 600 million school children are living with intestinal parasites. The prevalence of intestinal parasitic infections (IPIs) among school children in Ethiopia and the Amhara region is 52\% and 51\%, respectively. The present study aimed to determine the prevalence and associated risk factors of IPIs among primary school children in Dera district, Northwest Ethiopia. Methods. A school-based cross-sectional study was conducted from December 2019 to February 2020. The study used a structured pretested questionnaire and stool tests to obtain epidemiological and disease data. Data were analyzed using appropriate univariate and multivariable logistic regression methods by statistical package for social science (SPSS) version 25.0 . Results. Of the 382 students who were examined for IPIs, 238 (62.3\%) (61.8\% males, 62.8\% females) were positive for one or more IPIs. One hundred thirty-six (35.6\%), 98 (25.7\%), and 4 (1.05\%) were single, double, and triple infections, respectively. Out of the nine species of IPIs detected, Entamoeba sp. was the predominant species (29.6\%) followed by hookworm (21.7\%), Schistosoma mansoni (11.3\%), Taenia sp. (9.2\%), Giardia lamblia (5.2\%), and Ascaris lumbricoides, Hymenolepis nana, and Enterobius vermicularis (4.2\%) each, and Trichuris trichiura (0.5\%). Family size greater than five (AOR = 1.8; 95\% CI: 1.004, 3.13), open field school waste disposal $(\mathrm{AOR}=15.88 ; 95 \% \mathrm{CI}: 1.91,132.1)$, and lack of knowledge about intestinal parasitic infection $(\mathrm{AOR}=1.8$; 95\% CI: 1.1, 3.2) were the independent risk factors associated with the overall prevalence of IPIs. Conclusions. The prevalence of intestinal parasitic infection was high in the Dera district. Health education, extending school-based deworming, and mass treatments are recommended.
\end{abstract}

\section{Introduction}

Intestinal parasites are found in the gastrointestinal tracts of humans and other animals [1]. Globally, about 3.5 billion people are affected by parasitic infections. The annual morbidities and mortalities due to IPIs are estimated to be over 450 million and 200,000, respectively [2]. Morbidity and indirect effects of IPIs have a substantial impact on health and quality of life [3]. The World Health Organization (WHO) estimated that over 270 million preschool children and over 600 million school children are living in areas where the parasites are widely transmitted [3].
A. lumbricoides, T. trichiura, hookworms, H. nana, S. mansoni, E. histolytica/dispar, and G. lamblia are the major common IPIs reported globally $[1,3,4]$. The global trends of IPIs are 1.5 billion people with worms [5], 48 million people with amoeba, and 2.8 million people with giardia. The poorest and most deprived communities are highly affected [3].

In Ethiopia, 81 million people live in areas vulnerable to intestinal parasites, among which 25.3 million are school children (SC) [6]. Similarly, Ethiopia is a country with the lowest quality of drinking water supply (34\%) and latrine coverage $(7.1 \%)$ in the world [7]. Of the $7.1 \%$ latrine coverage, the latrine utilization level in Ethiopia is 50.02\% [8]. 
Consequently, intestinal parasites and other communicable diseases are widely distributed in various localities [7]. As a result, intestinal parasites are common in most parts of Ethiopia, particularly in school children, and can reach up to 84.3\% in some regions [9]. Studies in Ethiopia show the different prevalence of IPIs. For instance, the highest overall prevalence of IPIs is $84.3 \%$ in Debre Elias Primary Schools (North West Ethiopia) [9], and the lowest prevalence is from Babile town (13.8\%) (Eastern Ethiopia) [10].

IPIs are regarded as serious public health problems as they can cause iron deficiency anemia, growth retardation in children, and other physical and mental health conditions [11]. Furthermore, they cause abdominal pain, cholangitis, obstructive jaundice, acute pancreatitis, hepatic abscess, central nervous system disorders, ocular disorders, and epilepsy [12, 13], and death in more extreme cases [14]. Infections with intestinal parasites cause gastrointestinal problems such as diarrhea, dysentery, vomiting, and lack of appetite [15]. Diarrhea, including that of parasitic origin, is among the most common illnesses which cause infant and childhood mortality in developing countries [16].

Age is one of the risk factors for infection by intestinal parasites. School children are at a high risk of intestinal parasite infection. Poor hygiene, low immune status, overcrowding, close contact with soil and with each other, lack of latrine, and inadequate provision of safe water in schools are associated risk factors for IPIs [17-19]. Poverty, poor environmental hygiene, and impoverished health services are some of the other factors that put school children at high risk for IPIs [11].

According to clinical reports from a health center in Dera district (Northwest Ethiopia), intestinal infections are among the top reasons why people visit health facilities (Dera Woreda Health Office Annual Report, 2019). Among these, children are the dominant group infected by IPIs. However, there was no previous study conducted on the prevalence and associated risk factors of IPIs among school children in the current study area. Therefore, the present study aimed to assess the prevalence of IPIs and their risk factors among school children in the primary schools of Dera district, Northwest Ethiopia.

\section{Materials and Methods}

2.1. Study Area. This study was carried out in five primary schools from Dera district, South Gondar Zone, Amhara region, Northwest Ethiopia (Figure 1). It is located $535 \mathrm{~km}$ northwest of Addis Ababa (the capital city of Ethiopia) and $42 \mathrm{~km}$ away from Bahir Dar. Its geographical location is $11^{\circ}$ $43^{\prime} 0^{\prime \prime} \mathrm{N}$ and $37^{\circ} 38^{\prime} 0^{\prime \prime} \mathrm{E}$ and its elevation ranges from 1,560 to $2,600 \mathrm{~m}$ above sea level. Its mean annual temperature and rainfall are $26^{\circ} \mathrm{C}$ and $1250 \mathrm{~mm}$, respectively. Agroecologically, $85 \%$ of the district is lowland and the remaining $15 \%$ highland. Based on the 2007 projected national housing and population census, the total population of the district was 248,464 (126,961 males, 121,503 females). The majority of the population (93.25\%) lived in rural and $6.75 \%$ in urban areas. The main economic activity of the people in this area was agriculture [20].
There are 11 health centers and 36 health posts in the district. Health posts are assigned to implement the health extension program. Health Extension Workers (HEWs) spend $50 \%$ of their time visiting families in their homes and performing outreach activities in the community. HEWs are well-trained to provide first aid; treat diseases like malaria, dysentery, IPIs, and other ailments; and refer complicated cases to the nearest health center [21]. The district is rich with surface and groundwater resources such as Lake Tana and Abay River. However, access to clean water is limited. Thus, the community depends on various unprotected water sources.

2.2. Study Design. A school-based cross-sectional study was conducted from December 2019 to February 2020 to determine the prevalence of IPIs and associated risk factors among primary school students of Dera district, Northwest Ethiopia.

2.3. Study Population. The study population was all students enrolled in the five selected primary schools (from grades 1 to 8) $2019 / 2020$ academic year. The number of students in each school was as follows: Gibtsawit (761), Enbosa Maseria (685), Wagira (752), Mirafe Mariam (1318), and Korata (1277), which gives the total number of 4793 (2455 males, 2338 females).

2.4. Sample Size Determination. The sample size was determined using the single population formula $n=\left(Z^{2} p(1-p) / d^{2}\right)[22]$. The estimation of the sample size was based on population and previous studies on the prevalence of IPIs in the study area, where $n=$ sample size; $Z=1.96$ at $95 \%$ confidence level; $P=$ estimated prevalence rate $50 \%=0.5$, and $d=$ margin of error 0.05 . Since there was no similar study previously conducted in the area, a $50 \%$ prevalence rate of IPIs was taken assuming that IPIs are significantly prevalent among students in Gibtsawit, Mirafe Mariam, Wagira, Korata, and Enbosa Maseria primary schools. Accordingly, the total sample size was

$$
n=\frac{1.96^{2} 0.50(1-0.50)}{0.05^{2}}=384 \text { students. }
$$

The researchers proportionally selected 384 study participants from the five schools. The sample distribution per each school was Gibtsawit (61), Korata (102), Wagira (60), Mirafe Mariam (106), and Enbosa Maseria (55).

2.5. Sampling Methods. The five target schools were randomly selected from the 114 schools in the Dera district and the 384 students were selected using a simple random sampling method. The alphabetically arranged list of all students from the five schools (grades 1-8) served as a sampling frame. The respondents were grouped into three age categories 6 to 11 years (middle childhood), 12 to 18 years (early adolescence), and 19 to 21 years (late adolescence) [23]. 


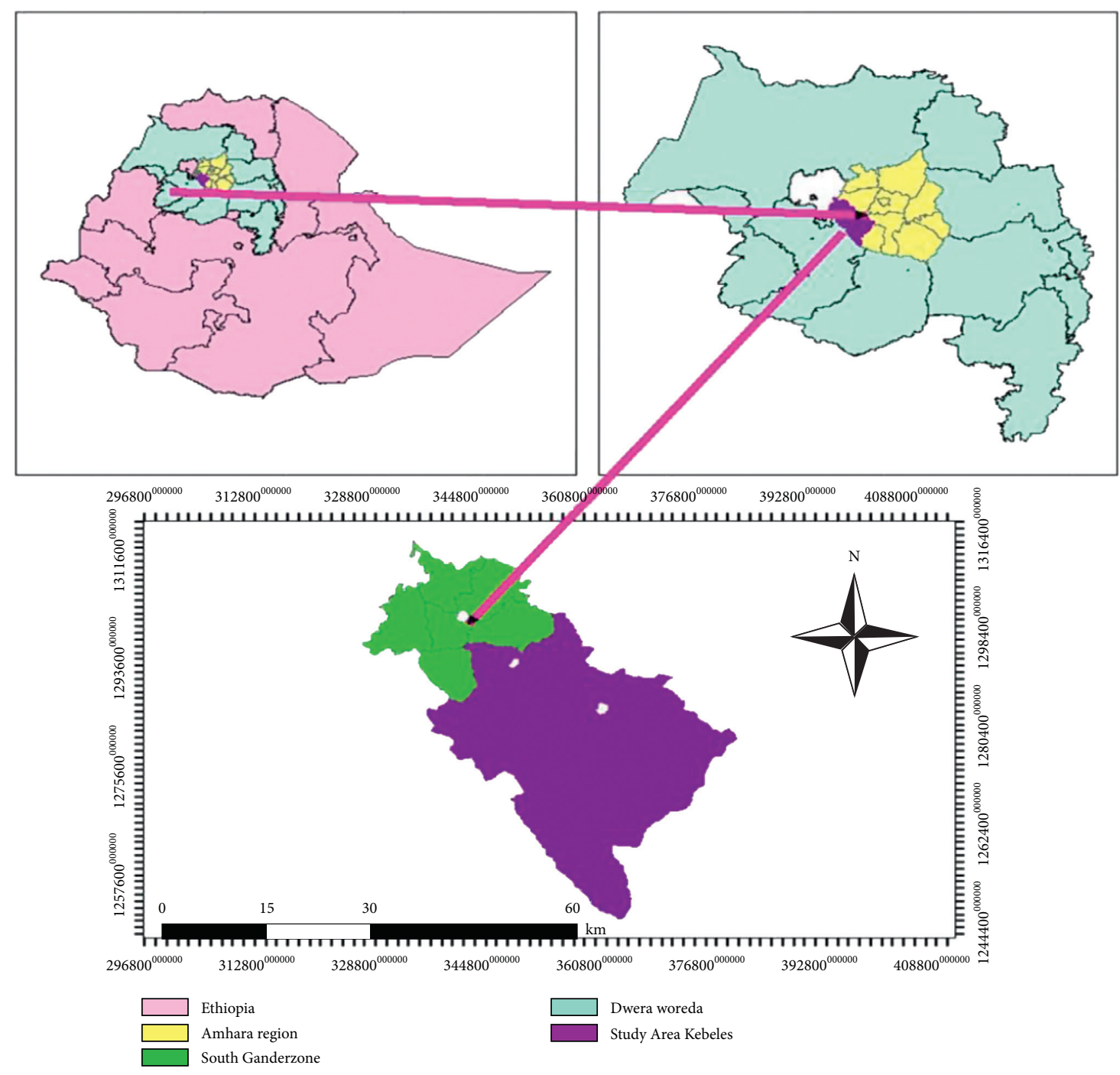

FIgURE 1: Location map of the study area.

2.6. Questionnaire Survey. Trained data collectors collected the information (sociodemographic characteristics, child behavioral characteristics, and past medical history) of every participant using interviews of structured questionnaires first developed in Amharic language and translated back to English while encoding the data. Data collectors also made clarifications of ambiguous questionnaires to children. The questionnaires were pretested and validated. The data collectors also checked the fingernail cleanness and the shoewearing habit of each student. The criteria for clean hands were properly trimmed fingernails, physically clean hands during observation, and handwashing habits after touching any dirt material and before eating. The data collectors considered the students knowledgeable about IPIs if they correctly mentioned at least two IPIs with their etiologic agents, modes of transmission, and prevention methods.

2.7. Stool Sample Collection. Formed and semi-formed fresh stool samples were used for parasitological analysis. The children were trained on how to collect the stool samples hygienically and advised to bring their own 3 to $5 \mathrm{~g}$ fresh stools in labeled collection cups along with applicator sticks. Parents were engaged for children aged 6-11.

2.8. Stool Sample Examination. Stool samples were examined under wet-mount and formol-ether concentration techniques. A portion of each of the stool samples was processed and examined microscopically using direct wetmount and formol-ether concentration techniques based on the procedures in WHO guidelines [24]. All developmental stages of the parasites (cyst, egg, larvae, and adult) were recorded.

2.9. Direct Microscopy (Wet Mount). A direct wet-mount technique was processed by conventional iodine. About $2 \mathrm{~g}$ of each stool sample was emulsified with 3-4 ml normal saline $(0.85 \% \mathrm{NaCl}$ solution). Then a drop of the emulsified sample was placed on a clean microscopic glass slide to observe the presence of trophozoites under the light microscope at $10 \mathrm{X}$ and $40 \mathrm{X}$. Then, a few drops of iodine 
solution were added to samples on glass slides and were covered with a coverslip. The presence of IPIs larvae, ova, and cysts was observed under the light microscope at $10 \mathrm{X}$ and 40X magnifications [24].

2.10. Formol-Ether Concentration Technique. A portion of each stool sample was used for the detection of parasitic ova and protozoan cysts using the formol-ether concentration technique based on the WHO guideline [24]. One gram (1 g) of each stool sample was first emulsified with 3-4 $\mathrm{ml}$ of $10 \%$ formalin saline. An additional 3-4 $\mathrm{ml}$ of $10 \%$ formol saline was added and mixed thoroughly and passed through gauze. Three to four (3-4) $\mathrm{ml}$ of diethyl ether was added and mixed by inverting and intermittent shaking of test tubes for 1 minute and centrifuged at 3,000 rpm for 5 minutes. After centrifugation, the layers of fecal debris were detached from the side of the tube using an applicator stick. The fecal debris and the formol saline were discarded and the sediment remained at the bottom of the test tube. The sediment was then transferred to a slide and covered with a coverslip. The preparation was examined microscopically using the $10 \mathrm{X}$ and 40X objectives for the identification of cysts and eggs.

2.11. Data Analysis. Statistical package for social science (SPSS) version 25.0 (IBM SPSS Statistics) was used to analyze the collected data. Chi-square $\left(\chi^{2}\right)$ test was performed to verify the possible association between the prevalence of IPIs and sociodemographic characteristics, socioeconomic characteristics, behavioral factors, personal hygiene, and environmental sanitation factors. Logistic regression was used to measure the degrees of association between the prevalence of IPIs and their associated risk factors. In the modeling process, univariate analysis was first done with a 0.25 level of significance to select the candidate variables for multivariable analysis. The variables, with a $P$ value of less than 0.25 , were qualified and included in the multivariable analysis [25]. A $P$ value below 0.05 was considered significant and 95\% CI was used to show the accuracy of data analysis.

2.12. Ethical Considerations. Before conducting the investigation, the investigator obtained ethical clearance from the Ethical Committee of Science College, Bahir Dar University (S1_File. Pdf). Written consents were obtained from the parents/guardians of children after explaining the purpose and the procedures of the study. The laboratory test was conducted with strict privacy and confidentiality. Finally, albendazole, mebendazole, and praziquantel drugs were given to the students whose test results were positive by the nurses free of charge.

2.13. Data Quality Control. The questionnaire was first pretested using thirty-five individuals outside the study population in other schools to avoid all ambiguities. All ambiguities were corrected before the questionnaires were administered to the actual study participants (S2_File. Pdf). During questionnaire surveys, the principal investigator cross-checked every questionnaire to check whether it was correctly filled by data collectors or not. The effectiveness of the reagents (formalin, diethyl ether, normal saline, and iodine solution) was pretested before starting the diagnosis of the study subjects by the laboratory technicians. A parasitological Atlas was consulted to ensure the accurate identification of the parasite species [26].

\section{Results}

3.1. Sociodemographic Characteristics of Study Participants in Dera District 2019/2020. Table 1 depicts the sociodemographic characteristics of the study participants. Of the 384 randomly identified participants, two students $(0.5 \%)$ were not voluntary to give stool samples and did not fill the structured questionnaire. Hence, 382 (99.5\%) (50\% male, $50 \%$ female) students participated in this study (response rate of $99.5 \%$ ). The age of the participants ranged from 6 to 21 years. Two hundred fifty-four $(66.5 \%)$ individuals fall in the age group 12 to 18 years, $123(32.2 \%)$ in 6 to 11 years, and five $(1.3 \%)$ in 19 to 21 years. Three hundred eight participants $(80.63 \%)$ were from family sizes of more than five and $74(19.37 \%)$ from less than or equal to five. The water sources of $67.8 \%$ of the students were from unprotected sources like springs, wells, and rivers. More than half (50.8\%) of the students did not wear shoes.

3.2. Prevalence of IPIs. Of the 382 students examined for IPIs, 238 (62.3\%; 95\% CI: 54.6, 70.7) were positive for one or more IPIs. The gender distribution of IPI positives was 118 (61.8\%; 95\% CI: 51.14, 73.98) males and 120 (62.8\%; 95\% CI: $52.09,75.13)$ females. The single, double, and triple IPIs were (136, 35.6\%; 95\% CI: 29.9, 42.11), (98, 25.7\%; 95\% CI: 20.83, $31.26)$, and (4, 1.05\%; 95\% CI: 0.29, 2.68), respectively. Nine parasites were detected using wet-mount and formol-ether concentration techniques (Table 2). Entamoeba sp. was the predominant species $(113,29.6 \%)$ followed by hookworm (83, 21.7\%), S. mansoni (43, 11.3\%), Taenia sp. (35, 9.2\%), G. lamblia $(20,5.2 \%), A$. lumbricoides, $H$. nana, E. vermicularis (16, 4.2\% each), and T. trichiura (2, 0.5\%). The prevalence of protozoan and helminths parasitic infections in the study area was $126(33 \%)$ and 171 (44.76\%), respectively.

3.3. Univariate and Multivariate Logistic Regression Analysis (LRA) of the Most Important Risk Factors for IPIs. The most important risk factors for IPIs among primary school children in the Dera district were identified using univariate and multivariate logistic regression analyses (MLRA) (Table 3).

Univariate logistic regression analyses showed that statistical differences in IPIs resulted due to variations in school, family size, modes of school waste disposal, knowledge of IPIs, playing with soil, suckling fingers, school toilet function, school water access, and household toilet $(P<0.25)$. The odds of IPIs were higher in Korata Primary School $(\mathrm{COR}=3.13$; 95\% CI: $1.544,6.344)$ than others, in students from schools openly disposing of wastes 
TABle 1: Sociodemographic characteristics of students $(n=382)$ from five primary schools (Gibtsawit Primary school, Enbosa Maseria, Wagira, Mirafe Mariam, and Korata Primary school) in Dera district, Northwest Ethiopia (2020).

\begin{tabular}{|c|c|c|c|}
\hline Sociodemographic variable & Categories & Frequency & Percent \\
\hline \multirow{5}{*}{ Schools } & Gibtsawit Primary School & 60 & 15.7 \\
\hline & Enbosa Maseria & 50 & 13.1 \\
\hline & Wagira & 58 & 15.2 \\
\hline & Mirafe Mariam & 109 & 28.5 \\
\hline & Korata Primary School & 105 & 27.5 \\
\hline \multirow{2}{*}{ Gender } & Male & 191 & 50 \\
\hline & Female & 191 & 50 \\
\hline \multirow{3}{*}{ Age (year) } & $6-11$ & 123 & 32.2 \\
\hline & $12-18$ & 254 & 66.5 \\
\hline & $19-21$ & 5 & 1.3 \\
\hline \multirow{2}{*}{ Grade level } & $1-4$ & 162 & 42.4 \\
\hline & $5-8$ & 220 & 57.6 \\
\hline \multirow{3}{*}{ Fathers' occupation } & Farmer & 379 & 99.2 \\
\hline & Merchant & 0 & 0 \\
\hline & Government employee & 3 & 0.8 \\
\hline \multirow{4}{*}{ Mothers' occupation } & Farmer & 311 & 81.4 \\
\hline & Merchant & 1 & 0.3 \\
\hline & Government employee & 3 & 0.8 \\
\hline & Housewife & 67 & 17.5 \\
\hline \multirow{5}{*}{ Maternal education } & Illiterate & 320 & 83.8 \\
\hline & Primary school & 14 & 3.7 \\
\hline & High school & 2 & 0.5 \\
\hline & Above high school & 3 & 0.8 \\
\hline & Adult education & 43 & 11.3 \\
\hline \multirow{5}{*}{ Paternal education } & Illiterate & 197 & 51.6 \\
\hline & Primary school & 32 & 8.4 \\
\hline & High school & 2 & 0.5 \\
\hline & Above high school & 5 & 1.3 \\
\hline & Adult education & 146 & 38.2 \\
\hline \multirow{2}{*}{ Toilet access } & No & 230 & 60.2 \\
\hline & Yes & 152 & 39.8 \\
\hline \multirow{4}{*}{ Household source of drinking water } & Spring & 52 & 13.6 \\
\hline & River & 30 & 7.9 \\
\hline & Well & 177 & 46.3 \\
\hline & Hand-dug well & 123 & 32.2 \\
\hline \multirow{2}{*}{ Personal hygiene } & Clean & 133 & 34.8 \\
\hline & Not clean & 249 & 65.2 \\
\hline \multirow{2}{*}{ Knowledge of IPI } & Yes & 295 & 77.2 \\
\hline & No & 87 & 22.8 \\
\hline \multirow{2}{*}{ Family size } & $\leq 5$ & 69 & 18.1 \\
\hline & $>5$ & 313 & 81.9 \\
\hline \multirow{2}{*}{ The habit of handwashing before a meal } & No & 14 & 3.7 \\
\hline & Yes & 368 & 96.3 \\
\hline \multirow{2}{*}{ The habit of shoe-wearing } & No & 194 & 50.8 \\
\hline & Yes & 188 & 49.2 \\
\hline
\end{tabular}

$(\mathrm{COR}=2.167 ; 95 \% \mathrm{CI}: 1.39,3.375)$ than burying/burning, among students lack IPI knowledge $(\mathrm{COR}=2.245 ; 95 \% \mathrm{CI}$ : $1.371,3.68)$ than those who knew, and among students who had school water access $(\mathrm{COR}=2.112 ; 95 \mathrm{CI}: 1.283,3.5)$ than not.

In the multivariate regression, family size more than five, open school waste disposal, and lack of knowledge were more likely associated with IPIs $(P<0.05)$. Students from the family size above five $(\mathrm{AOR}=1.8 ; 95 \% \mathrm{CI}: 1.004,3.13)$ were more likely to be infected by IPIs than those from five and below, students from schools where wastes were openly disposed of $(A O R=15.88 ; 95 \% \mathrm{CI}: 1.91,132.1)$ were prone to IPIs compared to those from schools which bury/burn wastes, and students who had no IPI knowledge were subjected to IPI $(\mathrm{AOR}=1.8 ; 95 \% \mathrm{CI}: 1.1,3.2)$ more than those known. 


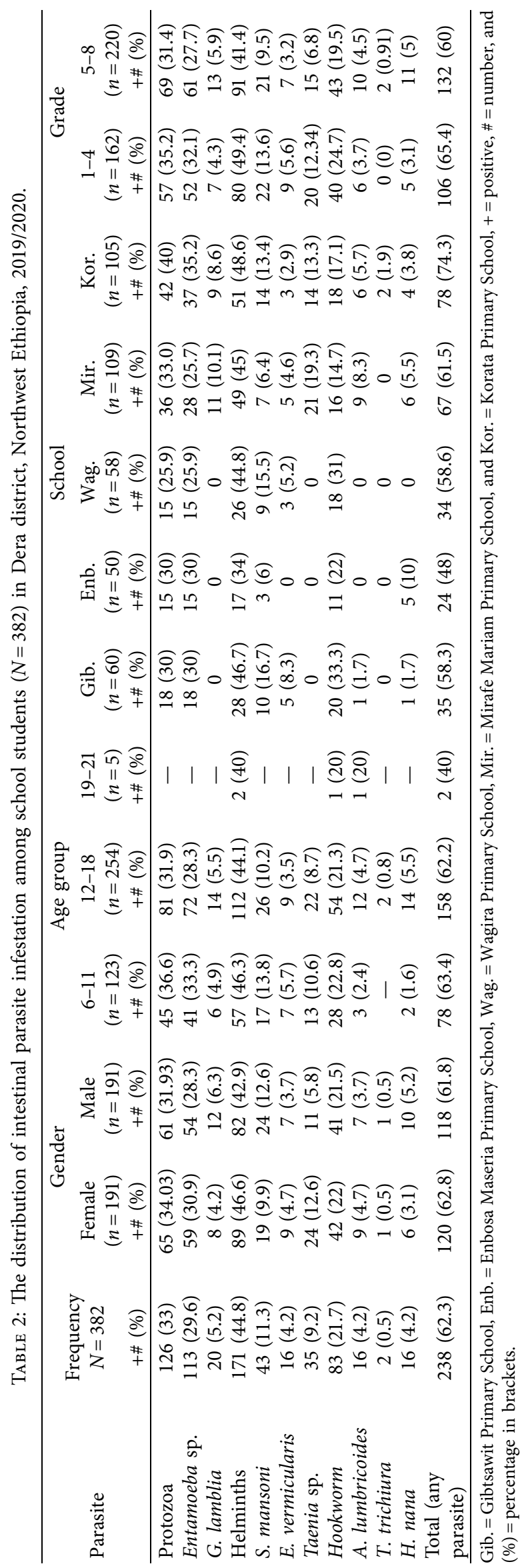


TABLE 3: Univariate and multivariate logistic regression analysis of potential risk factors associated with parasitic infection among school children in Dera district, Northwest Ethiopia, 2019/2020.

\begin{tabular}{|c|c|c|c|c|c|c|c|c|}
\hline \multirow[b]{2}{*}{ Risk factors } & \multirow[b]{2}{*}{ Categories } & \multicolumn{7}{|c|}{ IPIs } \\
\hline & & $\begin{array}{l}\text { Total no. } \\
\quad(\%)\end{array}$ & $\begin{array}{c}\text { Negative no. } \\
(\%)\end{array}$ & $\begin{array}{l}\text { Positive no. } \\
\text { (\%) }\end{array}$ & COR, $95 \% \mathrm{CI}$ & $\begin{array}{c}P \\
\text { value }\end{array}$ & $\mathrm{AOR}, 95 \% \mathrm{CI}$ & $\begin{array}{c}P \\
\text { value }\end{array}$ \\
\hline \multirow{5}{*}{ School } & $\begin{array}{c}\text { Gibtsawit Primary } \\
\text { School }\end{array}$ & $60(15.7)$ & $25(41.7)$ & $35(58.3)$ & $\begin{array}{c}1.52(0.712, \\
3.23)\end{array}$ & \multirow{5}{*}{$0.024^{*}$} & $\begin{array}{l}1.35(0.602 \\
3.028)\end{array}$ & \multirow{5}{*}{0.11} \\
\hline & $\begin{array}{l}\text { Enbosa Maseria } \\
\text { Primary School }\end{array}$ & $50(13.1)$ & $26(52)$ & $24(48)$ & 1 & & 1 & \\
\hline & $\begin{array}{c}\text { Wagira Primary } \\
\text { School }\end{array}$ & $58(15.2)$ & $24(41.4)$ & $34(58.6)$ & $\begin{array}{c}1.535(0.716 \\
3.289)\end{array}$ & & $\begin{array}{c}0.112(0.012 \\
1.1)\end{array}$ & \\
\hline & $\begin{array}{l}\text { Mirafe Mariam } \\
\text { Primary School }\end{array}$ & $109(28.5)$ & $42(38.5)$ & $67(61.5)$ & $1.73(0.9,3.4)$ & & $\begin{array}{c}0.096(0.01 \\
0.8)\end{array}$ & \\
\hline & Korata Primary School & $105(27.5)$ & $27(25.7)$ & $78(74.3)$ & $\begin{array}{c}3.13(1.544, \\
6.344) \\
\end{array}$ & & - & \\
\hline \multirow[b]{2}{*}{ Family size } & $\leq 5$ & $69(18.1)$ & $34(49.3)$ & $35(50.7)$ & 1 & \multirow[b]{2}{*}{$0.030^{*}$} & 1 & \multirow[b]{2}{*}{$0.049^{*}$} \\
\hline & $>5$ & $313(81.9)$ & $110(35.1)$ & $203(64.9)$ & $1.8(1.1,3.03)$ & & $\begin{array}{c}1.8(1.004, \\
3.13)\end{array}$ & \\
\hline \multirow{2}{*}{$\begin{array}{l}\text { Mode of school waste } \\
\text { disposal }\end{array}$} & Burry and firing & $119(31.2)$ & $60(50.4)$ & $59(49.6)$ & \multirow{2}{*}{$\begin{array}{c}1 \\
2.167(1.39 \\
3.375)\end{array}$} & \multirow[b]{2}{*}{$0.001^{*}$} & 1 & \multirow[b]{2}{*}{$0.011^{*}$} \\
\hline & Open field & $263(68.8)$ & $84(31.9)$ & $179(68.1)$ & & & $\begin{array}{c}15.88(1.91 \\
132.1)\end{array}$ & \\
\hline \multirow[t]{2}{*}{ Knowledge of IPIs } & Yes & $299(78.3)$ & $100(33.9)$ & $199(67.5)$ & $\begin{array}{c}2.245(1.371 \\
3.68)\end{array}$ & \multirow[t]{2}{*}{$0.001^{*}$} & $1.8(1.1,3.2)$ & \multirow[t]{2}{*}{$0.027^{*}$} \\
\hline & No & $83(21.7)$ & $44(50.6)$ & $39(44.8)$ & 1 & & 1 & \\
\hline \multirow[b]{2}{*}{ Playing with soil } & No & $208(54.5)$ & $84(40.4)$ & $124(59.6)$ & \multirow{2}{*}{$\begin{array}{c}1 \\
1.287(0.85 \\
1.95)\end{array}$} & \multirow[b]{2}{*}{$0.24^{*}$} & 1 & \multirow[b]{2}{*}{0.148} \\
\hline & Yes & $174(45.5)$ & $60(34.5)$ & $114(65.5)$ & & & $\begin{array}{l}1.5(0.87 \\
2.45)\end{array}$ & \\
\hline \multirow[b]{2}{*}{ Suckling's fingers } & No & $130(34.0)$ & $55(42.3)$ & $75(57.7)$ & \multirow{2}{*}{$\begin{array}{c}1 \\
1.343(0.871 \\
2.07)\end{array}$} & \multirow[b]{2}{*}{$0.18^{*}$} & 1 & \multirow[b]{2}{*}{0.963} \\
\hline & Yes & $252(66.0)$ & $89(35.3)$ & $163(64.7)$ & & & $\begin{array}{c}1.01(0.59 \\
1.7)\end{array}$ & \\
\hline \multirow[t]{2}{*}{ School toilet function } & No & $188(49.2)$ & $57(30.3)$ & $131(69.7)$ & $\begin{array}{l}1.869(1.23 \\
2.845)\end{array}$ & \multirow[t]{2}{*}{$0.004^{*}$} & $\begin{array}{l}1.4(0.825 \\
2.225)\end{array}$ & \multirow[t]{2}{*}{0.230} \\
\hline & Yes & $194(50.8)$ & $87(44.8)$ & $107(55.2)$ & 1 & & 1 & \\
\hline \multirow[t]{2}{*}{ School water access } & No & $105(27.5)$ & $27(25.7)$ & $78(74.3)$ & $\begin{array}{c}2.112(1.283 \\
3.5)\end{array}$ & \multirow[t]{2}{*}{$0.003^{*}$} & $5.3(0.55,50)$ & \multirow[t]{2}{*}{0.146} \\
\hline & Yes & $277(72.5)$ & $117(42.2)$ & $160(57.8)$ & 1 & & 1 & \\
\hline \multirow[t]{2}{*}{ Household toilet } & No & $230(60.2)$ & $81(35.2)$ & $149(64.8)$ & $\begin{array}{c}1.302(0.9 \\
1.9)\end{array}$ & \multirow[t]{2}{*}{$0.219^{*}$} & $\begin{array}{l}1.4(0.9 \\
2.204)\end{array}$ & \multirow[t]{2}{*}{0.178} \\
\hline & Yes & $152(39.8)$ & $63(41.4)$ & $89(58.6)$ & 1 & & 1 & \\
\hline
\end{tabular}

*Statistically significant at $P<0.05 ; 1=$ reference value; $\mathrm{AOR}=$ adjusted odds ratio; $\mathrm{COR}=$ crude odds ratio.

3.4. Risk Factors Associated with Entamoeba sp., G. lamblia, E. vermicularis, Taenia sp., and A. lumbricoides. Entamoeba sp., G. lamblia, E. vermicularis, Taenia sp., and A. lumbricoides were the most frequent IPIs detected from the study participants. Their risk factors and association are presented in Table 4 .

3.5. Risk Factors Associated with Entamoeba sp. Infection. The multivariate regression analysis shows that drinking river water, eating soil, and lack of school water access were significantly associated with Entamoeba sp. infection $(P<0.05)$ (Table 4). Students who used to drink river water $(\mathrm{AOR}=2.35 ; 95 \% \mathrm{CI}: 1.102,5.866)$ were at high risk of Entamoeba sp. infection than those who used to drink handdug well water, students who ate soil $(\mathrm{AOR}=3.96$; $95 \% \mathrm{CI}$; $1.34,11.67)$ were more prone to Entamoeba sp. than their counterparts, and students from schools which lack water access $(\mathrm{AOR}=1.773 ; 95 \% \mathrm{CI}: 1.004,3.13)$ were more likely infected by Entamoeba sp. than students from schools where there is enough water.

3.6. Risk Factors Associated with G. lamblia Infection. Maternal handwashing habits and school toilet functions were the only predictors of $G$. lamblia in the multivariable analysis $(P<0.05)$ (Table 4$)$. Students from mothers who used to wash their hands with water alone, students from mothers who did not wash their hands, and students who did not use the school toilet were strongly associated with G. lamblia $(P<0.05)$. Students from mothers who used to wash their hands with water alone $(\mathrm{AOR}=7.973 ; 95 \% \mathrm{CI}$ : $0.97,64.4$ ) and students from mothers who did not wash their hands $(\mathrm{AOR}=2.1 ; 95 \% \mathrm{CI}: 0.17,25.1)$ were subjected to 
TABLE 4: Univariate and multivariate logistic regression analysis of potential risk factors associated with Entamoeba sp., G. lamblia, E. vermicularis, Taenia sp., and A. lumbricoides infection among school children in Dera district, Northwest Ethiopia.

\begin{tabular}{|c|c|c|c|c|c|c|c|c|}
\hline \multicolumn{9}{|c|}{ Entamoeba sp. } \\
\hline Risk factors & Categories & $\begin{array}{l}\text { Total no. } \\
\text { (\%) }\end{array}$ & $\begin{array}{c}\text { Negative no. } \\
(\%)\end{array}$ & $\begin{array}{l}\text { Positive } \\
\text { no. (\%) }\end{array}$ & COR, 95\% CI & $\begin{array}{c}P \\
\text { value }\end{array}$ & AOR, 95\% CI & $\begin{array}{c}P \\
\text { value }\end{array}$ \\
\hline \multirow{4}{*}{$\begin{array}{l}\text { Household source of drinking } \\
\text { water }\end{array}$} & River & $30(7.9)$ & $15(50)$ & $15(50)$ & $\begin{array}{l}2.417(1.07 \\
5.456)\end{array}$ & \multirow{3}{*}{$0.096^{*}$} & $\begin{array}{c}2.351(1.031 \\
5.360)\end{array}$ & \multirow{4}{*}{$0.039^{*}$} \\
\hline & Spring & $52(13.6)$ & $38(73.1)$ & $14(26.9)$ & $\begin{array}{c}0.89(0.431 \\
1.839)\end{array}$ & & $\begin{array}{c}0.835(0.395 \\
1.764)\end{array}$ & \\
\hline & Well & $177(46.3)$ & $129(72.9)$ & $48(27.1)$ & $\begin{array}{c}0.899(0.54 \\
1.498)\end{array}$ & & $\begin{array}{c}0.706(0.395 \\
1.260)\end{array}$ & \\
\hline & $\begin{array}{c}\text { Hand-dug } \\
\text { well }\end{array}$ & $123(32.2)$ & $87(70.7)$ & $36(29.3)$ & 1 & & 1 & \\
\hline \multirow[b]{2}{*}{ Eating soil } & No & $367(96.1)$ & $263(71.66)$ & $104(28.3)$ & 1 & & 1 & \multirow[b]{2}{*}{$0.013^{*}$} \\
\hline & Yes & $15(3.9)$ & $6(40)$ & $9(60)$ & $\begin{array}{c}3.79(1.317 \\
10.92)\end{array}$ & $0.013^{*}$ & $\begin{array}{c}3.957(1.34 \\
11.67) \\
\end{array}$ & \\
\hline \multirow[t]{2}{*}{ School water access } & No & $105(27.5)$ & $68(64.8)$ & $37(35.2)$ & $\begin{array}{l}1.439(0.89 \\
2.325)\end{array}$ & \multirow[t]{2}{*}{$0.137^{*}$} & $\begin{array}{c}1.773(1.004 \\
3.1)\end{array}$ & \multirow[t]{2}{*}{$0.048^{*}$} \\
\hline & Yes & $277(72.5)$ & $201(72.6)$ & $76(27.4)$ & 1 & & 1 & \\
\hline \multicolumn{9}{|c|}{ G. lamblia } \\
\hline \multirow{3}{*}{ Maternal handwashing } & No & $85(22.3)$ & 83 (97.6) & $2(2.4)$ & $\begin{array}{c}1.687(0.15, \\
18.99)\end{array}$ & \multirow{3}{*}{$0.083^{*}$} & $\begin{array}{c}2.09(.174, \\
25.1)\end{array}$ & \multirow{3}{*}{$0.042^{*}$} \\
\hline & $\begin{array}{c}\text { Yes only by } \\
\text { water }\end{array}$ & $226(59.2)$ & $209(92.5)$ & $17(7.5)$ & $\begin{array}{c}5.694(0.74, \\
43.56)\end{array}$ & & $\begin{array}{c}7.973(0.99 \\
64.5)\end{array}$ & \\
\hline & Yes by soap & $71(18.6)$ & $70(99)$ & $1(1)$ & 1 & & 1 & \\
\hline \multirow{2}{*}{$\begin{array}{l}\text { Taking an antiparasitic drug } \\
\text { before } 6 \text { months }\end{array}$} & No & $323(84.6)$ & $304(94.1)$ & $19(5.9)$ & $\begin{array}{c}3.625(0.476 \\
27.6)\end{array}$ & \multirow[t]{2}{*}{$0.214^{*}$} & $\begin{array}{c}3.14(.39 \\
25.21)\end{array}$ & \multirow[t]{2}{*}{0.282} \\
\hline & Yes & $59(15.4)$ & $58(98.3)$ & $1(1.7)$ & 1 & & 1 & \\
\hline \multirow[t]{2}{*}{ Knowledge of IPIs } & No & $299(78.3)$ & $281(94)$ & $18(6)$ & $\begin{array}{c}2.594(0.59, \\
11.42)\end{array}$ & $0.207^{*}$ & $\begin{array}{c}1.735(.37 \\
8.08)\end{array}$ & \multirow[t]{2}{*}{0.483} \\
\hline & Yes & $83(21.7)$ & $81(97.6)$ & $2(2.4)$ & 1 & & 1 & \\
\hline \multirow[t]{2}{*}{ School water access } & No & $105(27.5)$ & $96(91.4)$ & $9(8.6)$ & $\begin{array}{c}2.267(0.911 \\
5.64)\end{array}$ & $0.078^{*}$ & $\begin{array}{c}2.101(0.78 \\
5.59)\end{array}$ & \multirow[t]{2}{*}{0.137} \\
\hline & Yes & $277(72.5)$ & $266(96)$ & $11(4)$ & 1 & & 1 & \\
\hline \multirow[t]{2}{*}{ School toilet function } & No & $188(49.2)$ & $172(91.5)$ & $16(8.5)$ & $\begin{array}{c}4.419(1.45, \\
13.5)\end{array}$ & $0.009^{*}$ & $\begin{array}{c}4.132(1.3 \\
13.03)\end{array}$ & \multirow[t]{2}{*}{$0.015^{*}$} \\
\hline & Yes & $194(50.8)$ & $190(97.9)$ & $4(2.1)$ & 1 & & 1 & \\
\hline \multicolumn{9}{|c|}{ E. vermicularis } \\
\hline \multirow{3}{*}{ Maternal hand wash } & No & $85(22.3)$ & $84(98.8)$ & $1(1.2)$ & $\begin{array}{c}0.411(0.036 \\
4.63)\end{array}$ & & $\begin{array}{c}0.432(0.034 \\
5.6)\end{array}$ & \multirow{3}{*}{0.118} \\
\hline & $\begin{array}{l}\text { Yes only by } \\
\text { water }\end{array}$ & $226(59.2)$ & $213(94.2)$ & $13(5.8)$ & $\begin{array}{c}2.106(0.464 \\
9.56)\end{array}$ & $0.212^{*}$ & $\begin{array}{c}2.858(0.53 \\
15.5)\end{array}$ & \\
\hline & Yes by soap & $71(18.6)$ & $69(97.2)$ & $2(2.8)$ & 1 & & 1 & \\
\hline \multirow{4}{*}{ Household drinking water } & Boiling & $3(0.8)$ & $3(100)$ & $0(0)$ & $0.000(0.000)$ & & --------- & \multirow{4}{*}{$0.030^{*}$} \\
\hline & Filtering & $36(9.4)$ & $32(88.9)$ & $4(11.1)$ & $\begin{array}{c}1.250(0.209 \\
7.46)\end{array}$ & & $\begin{array}{c}1.378(0.19 \\
10.1)\end{array}$ & \\
\hline & Direct & $\begin{array}{c}321 \\
(84.03)\end{array}$ & $311(96.9)$ & $10(3.4)$ & $\begin{array}{c}0.322(0.07 \\
1.567)\end{array}$ & $0.125^{*}$ & $\begin{array}{c}0.206(0.038 \\
1.13)\end{array}$ & \\
\hline & $\begin{array}{c}\text { Chlorine } \\
\text { treated }\end{array}$ & $22(5.8)$ & $20(90.9)$ & $2(9.1)$ & 1 & & 1 & \\
\hline \multirow[t]{2}{*}{ Knowledge of IPIs } & No & $299(78.3)$ & $284(94.9)$ & $15(5.02)$ & $\begin{array}{c}4.331(0.56 \\
33.28)\end{array}$ & $0.159^{*}$ & $\begin{array}{c}5.473(0.67 \\
44.69)\end{array}$ & 0.113 \\
\hline & Yes & $83(21.7)$ & $82(99)$ & $1(1)$ & 1 & & 1 & \\
\hline & No & $147(38.5)$ & $144(97.9)$ & $3(2.1)$ & 1 & & 1 & \\
\hline A habit of eating raw meat & Yes & $235(61.5)$ & $222(94.5)$ & $13(5.5)$ & $\begin{array}{c}2.811(0.79 \\
10.04)\end{array}$ & $0.112^{*}$ & $\begin{array}{c}3.581(0.94 \\
13.7)\end{array}$ & 0.062 \\
\hline
\end{tabular}


TABLE 4: Continued.

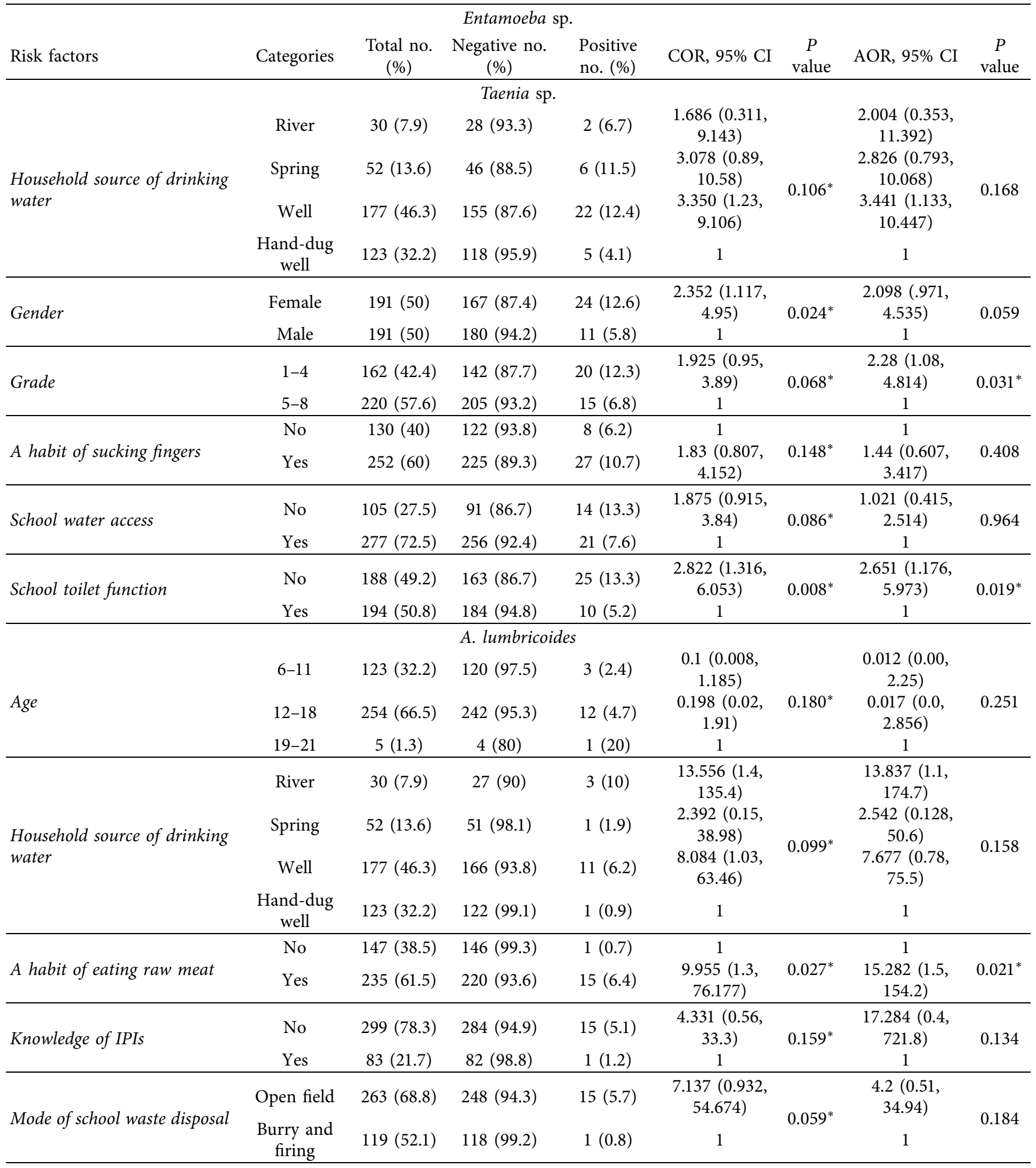

*Statistically significant at $P<0.05 ; 1=$ reference value; $\mathrm{AOR}=$ adjusted odds ratio; $\mathrm{COR}=$ crude odds ratio.

G. lamblia as compared to children from mothers who used to wash their hands with soap. Students who did not use the school toilet (AOR $=4.13,95 \%$ CI: $1.3,13.03)$ were prone to G. lamblia more than students who used to.
3.7. Risk Factors Associated with E. vermicularis Infection. The household drinking water source was the only risk factor associated with $E$. vermicularis $(P<0.05)$ in the multivariable analysis (Table 4). Students who used to drink locally 
filtered water $(\mathrm{AOR}=1.378$; 95\% CI: 0.188, 10.095) were more likely to be infected with $E$. vermicularis than students who used to drink chlorinated water.

3.8. Risk Factors Associated with Taenia sp. Infection. Statistically significant differences in Taenia sp. $(P<0.05)$ were seen with differences in grade levels and school toilet functions in the multivariate logistic regression analysis (Table 4). Grades 1-4 and not using the school toilet were associated with Taenia sp. infection. Students from grades $1-4(\mathrm{AOR}=2.28 ; 95 \% \mathrm{CI}: 1.08,4.814)$ were more likely to be infected by Taenia sp. than students from grades $5-8$ and students who did not use school toilet $(\mathrm{AOR}=2.651 ; 95 \% \mathrm{CI}$ : $1.176,5.973$ ) were more subjected to Taenia sp. than children who did use the school toilet.

3.9. Risk Factors Associated with A. lumbricoides Infection. Only the habit of eating raw meat resulted in statistically significant differences in A. lumbricoides infection $(P<0.05)$ (Table 4). Children who ate raw meat $(\mathrm{AOR}=15.282 ; 95 \%$ CI: $1.514,154.233)$ were more likely to be infected by A. lumbricoides than children who did not eat raw meat.

\section{Discussion}

The overall prevalence of IPIs in the present study was $62.3 \%$. It was comparable with the studies done in Sasiga District, Southwest Ethiopia (62.4\%) [27], Sudan (56.9\%) [28], Bahir Dar, Ethiopia (65.5\%) [29], Southern Ethiopia (56\%) [30], and Jawi town, North West Ethiopia (57.88\%) [19].

This overall prevalence was higher than that of the national prevalence of IPIs among school children (52\%) [31], Senbete and Bete Towns, North Shoa, Ethiopia (52.3\%) [32], Homesha District, Northwest Ethiopia (35.44\%) [33], Birbir town, Southern Ethiopia (27.1\%) [34], Delo-Mena District, Southeastern Ethiopia (26.6\%) [35], Adigrat town, Northern Ethiopia (50.81\%) [36], Gurage Zone, Ethiopia (40.2\%) [37], Bahir Dar, Ethiopia (45\%) [38], Gobgob Primary School, Northwest Ethiopia (30.8\%) [39], East Arsi, Ethiopia (27.1\%) [40], Iran (21.5\%) [18], India (27.5\%) [41], Western Saudi Arabia (12\%) [42], Nigeria (13.7\%) [43], Rwanda (44.8\%) [44], and Senegal (35\%) [45]. But, it was lower than the finding from Mizan-Aman Town, Southwest Ethiopia (76.7\%) [46]. These differences could be attributed to the differences in the socioeconomic status of the people, sociodemographic distinctions, knowledge, hygiene, and sanitary facilities, weather, climate, and environmental factors.

The prevalence of IPIs among the male and female students in the Dera District was $61.8 \%$ and $62.8 \%$, respectively. No statistically significant difference was observed between male and female students. The outcome of the study was in line with the studies done in Glomekeda district, Northern Ethiopia, males (31.3\%) and females (28.6\%) [47]; Sasiga District, Southwest Ethiopia, males (62.6\%) and females (62.2\%) [27]; Bahir Dar, Ethiopia, males $(67.8 \%)$ and females (63.2\%) [29]; Jawi Town, Ethiopia, males (51.85\%) and females (45.30\%) [19]; and Adigrat town, Northern Ethiopia, males (49.13\%) and females (52.94\%) [36], $P>0.05$.

However, it disagrees with the studies done in Gobgob Northwest, Ethiopia, where males (38.9\%) were more infected than females $(26.4 \%) P<0.05$ [39]. This equal chance of IPIs among males and females may be due to the narrowing of gender role differences in the study area.

Korata, Mirafe Mariam, and Wagira primary schools are found near Lake Tana. Their soil is wet, swampy, and suitable for agriculture and irrigation activities. The people from these areas are farmers and their children are engaged in farming and irrigation activities. Wagira has one dam that is used for irrigation cultivating vegetables, fruits, onions, khat, and cereals. Accordingly, the prevalence of IPIs was high in Korata (74.3\%), Mirafe Mariam (61.5\%), and Wagira (58.6\%).

The prevalence of IPIs in Korata $(74.3 \%)$ was relatively similar to the studies done in Mizan-Aman town, Ethiopia (72.9\%), Ediget Behibret, Ethiopia (88.9\%), and Mizan Number One, Ethiopia (83.4\%) [46]. The prevalence of IPIs in Mirafe Mariam (61.5\%) and Wagira (58.6\%) was close to the studies in Cambodia: Dontri locality (54.3\%) and Kon Kaêk locality (45.5\%) [48]. The result was higher than the studies done in Gurage Zone, Enemorena-Ener District, Ethiopia (40\%) [37], Gurage Zone, Abeshege Districts, Ethiopia (38.7\%) [37], Mizan-Aman town, Ethiopia (42.9\%), Mizan Misgana Acadamy, Ethiopia (35.5\%), and Aman Misgana Academy, Ethiopia (30.0\%) [46]. However, it was much lower than the study done in Mizan-Aman town, Gacheb School (90\%) [46].

The prevalence of protozoa parasitic infections in our study was $33 \%$. The outcome of the study was higher than the studies done in Birbir town, Southern Ethiopia (7.1\%) [34], Gobgob, Northwest Ethiopia (7.32\%) [39], Sasiga District, Southwest Ethiopia (6.8\%) [27], Adele town, East Arsi, Ethiopia (17.7\%) [40], Jawi town, Northwest Ethiopia (25.8\%) [19], Iran (21\%) [18], Sri Lanka (18.4\%) [49], and Adigrat town, Northern Ethiopia (6.8\%) [36].

On the contrary, the outcome was comparable with the studies conducted in Glomekeda District, Northern Ethiopia (27.8\%) [47], and Bahir Dar, Ethiopia (35.9\%) [29]. However, it was lower than the studies from Senegal (93.4\%) [45] and Sudan (54.2\%) [28]. The contradictory reports on the prevalence of protozoan infections could be due to variations in geography, socioeconomic conditions (unprotected source of drinking water, poor personal and environmental sanitation, and accessibility and use toilets), and the methods employed for stool examination, and the time of the study.

The prevalence of helminths in the study area was $44.76 \%$. The result was lower than the studies done in Malaysia (60.8\%) [50] and Sasiga District, Southwest Ethiopia (61.1\%) [27]. However, it was higher than the studies done in Sudan (2.80\%) [28], Iran (0.36\%) [18], Senegal (2.2\%) [45], Jawi Town, Northwest Ethiopia (32\%) [19], Babble town, Eastern Ethiopia (13.8\%) [10], Birbir town, Southern Ethiopia (25.4\%) [34], Glomekeda district, Northern Ethiopia (12.08\%) [47], Adele town, East Arsi, Ethiopia (26.4\%) [40], and Gobgob Northwest, Ethiopia 
(23.44\%) [39]. But, it was comparable to Bahir Dar, Ethiopia (50.14\%) [29], and Adigrat town, Northern Ethiopia (44.013\%) [36]. The variation could be because of differences in socioeconomic distinction, the habit of wearing shoes, participation in agricultural activities, the habit of swimming, and eating uncooked/raw vegetables and foods.

Nine species of IPIs were isolated from the study area. Entamoeba sp. (29.6\%) was the most predominant parasite followed by hookworm (21.7\%), S. mansoni (11.3\%), Taenia sp. (9.2\%), G. lamblia (5.2\%), A. lumbricoides, H. nana, and E. vermicularis (4.2\% each), and T. trichiura (0.5\%) (Table 2).

The prevalence of Entamoeba sp. (29.6\%) in the present study was relatively similar to the studies conducted in Bahir Dar, Ethiopia (24.5\%) [29], Tanzania (31\%) [51], Sudan, (31.2\%) [28], and Rwanda (25.95\%) [44]. However, it was higher than the study done in Gobgob, Northwest Ethiopia (13\%) [39], Sudan (15.50\%) [17], Homesha District, Western Ethiopia (14.17\%) [33], Jawi town, Ethiopia (5.9\%) [19], Cambodia (17.5\%) [48], Birbir town, Southern Ethiopia (2.6\%) [39], Delo-Mena District, Southeastern Ethiopia (7.7\%) [35], Sasiga District, Southwest Ethiopia (8.1\%) [27], Adigrat town, Northern Ethiopia (4.5\%) [36], Glomekeda district, Northern Ethiopia (19.43\%) [47], Adele town, East Arsi, Ethiopia (10.3\%) [40], and western Saudi Arabia (2\%) [42]. But, it was lower than the study conducted in Nigeria (39.5\%) [43]. The difference in the prevalence of Entamoeba sp. among the different studies might be due to the differences in the levels of contamination of drinking water sources, consumption of raw vegetables, and handwashing habits of the study participants [52].

The prevalence of G. lamblia (5.2\%) in the study area was lower than the studies done in Bahir Dar Ethiopia (11.4\%) [29], Homesha District, Northwest Ethiopia (12.65\%) [33], Jawi town, Ethiopia (19.95\%) [19], Gobgob, Northwest Ethiopia (11\%) [39], Iran (11.0\%) [53], Nigeria (19.7\%) [43], Rwanda (19.6\%) [44], Sudan (22.9\%) [28], Tripoli, Lebanon (28.5\%) [54], Cambodia (31.5\%) [48], and Senegal (72.5\%) [45].

On the other hand, G. lamblia prevalence (5.2\%) was relatively close to the findings in Delo-Mena (2.0\%) [35], Adigrat town, Northern Ethiopia (2.29\%) [36], Sasiga District, Southwest Ethiopia (6.5\%) [27], Birbir town, Southern Ethiopia (4.8\%) [34], Glomekeda district, Northern Ethiopia, (8.29\%) [47], and western Saudi Arabia (3\%) [42]. But, it was higher than the study from Iran (1.66\%) [18]. These variations might be due to variations in the quality of drinking water sources, foods, utilization of toilets, washing hands, and environmental conditions.

The prevalence of hookworm in the study area was $21.7 \%$. This result was relatively similar to the studies done in Bahir Dar, Ethiopia (22.8\%) [29], Birbir town, Southern Ethiopia (19.54\%) [34], Sasiga District, Southwest Ethiopia (20.6\%) [27], and Nigeria (19.7\%) [43]. The outcome of this study was higher than in Delo-Mena district (0.8\%) [35], Dera Woreda, Ethiopia (14.7\%) [55], Homesha District, Northwest Ethiopia (10.12\%) [33], Jawi town, Ethiopia (13.8\%) [19], Gobgob, Northwest Ethiopia (8.3\%) [39], Glomekeda district, Northern Ethiopia (0.7) [47], and Malaysia (4.1\%) [50]. But, it was lower than the study done in
Nigeria (45.62\%) [56], and Bahir Dar, Ethiopia (54.5\%) [57]. These reported variations in the prevalence of hookworm infection among studies could be attributed to differences in environmental sanitation, mass treatment, school deworming, and shoes wearing habits of the students.

The prevalence of $S$. mansoni in the study area was $11.3 \%$. The outcome was relatively similar to the outcome of the Delo-Mena district (9.6\%) [35], Jawi town, Ethiopia (10.3\%) [19], and Bahir Dar Zuria, Ethiopia (15.7\%) [57]. However, it was higher than the studies done in Gurage Zone, Ethiopia (1.7\%) [37], Homesha District, Northwest Ethiopia (0.75\%) [33], Sudan (2.40\%) [17], Birbir town, Southern Ethiopia (1.1\%) [34], and Sasiga District, Southwest Ethiopia (4.4\%) [27]. The possible reason for the high prevalence of $S$. mansoni infection in the present study might be that children have contact with river water during bathing, swimming, fetching water for home use, and irrigation activities. The study area is known for irrigation activities and children participate in agricultural activities to help their parents. The district has rivers (Gumara and Gelda), Lake Tana, Wagira Dam, and other smallest springs used as water sources.

The prevalence of Taenia sp. (9.2\%) was in line with the studies done in Nigeria (5.3\%) [43], Malaysia (9.5\%) [50], and Gobgob, Northwest Ethiopia (7\%) [39]. However, it was higher than that of Delo-Mena district, Ethiopia (0.2\%) [35], Gurage Zone, Ethiopia (1.6\%) [37], Homesha District, Northwest Ethiopia (1.77\%) [33], and Jawi town, Ethiopia (3\%) [19]. Consumption of raw meat and vegetables, drinking water from unprotected water sources, playing with soil, grade level, presence of domestic animals, the habit of sucking fingers, the habit of open defecation, and participating in agriculture activities might be reasons for Taenia sp. infection.

Prevalence of $A$. lumbricoides (4.2\%) was similar to the studies done in Nigeria (7.9\%) [44], Delo-Mena district, Ethiopia (3.7\%) [35], Gurage Zone, Ethiopia (9.4\%) [37], Birbir town, Southern Ethiopia (8.8\%) [34], Bahir Dar Zuria, Ethiopia (8.6\%) [57], and Glomekeda district, Northern Ethiopia (3.3\%) [47]. The result was lower than the study conducted in Bahir Dar, Ethiopia (13.6\%) [29], Nigeria (26.8\%) [56], Malaysia (34.3\%) [50], Gobgob Northwest Ethiopia (33.3\%) [39], Southern Ethiopia (33.2\%) [30], Sasiga District, Southwest Ethiopia (22.7\%) [27], Adigrat town, Northern Ethiopia (19.1\%) [36], Adele town, East Arsi, Ethiopia (12\%) [40], and Southwestern China (25.2\%) [58]. But, it was higher than studies from Homesha District, Northwest Ethiopia (0.5\%) [33], Jawi town, Ethiopia (0.73\%) [19], and Sudan (1.20\%) [17]. The possible reason for these differences might be explained due to differences in eating raw vegetables, playing with soil, not washing hands before food, and after latrine, participation in agricultural activities, knowledge of IPIs, school waste disposal habit, drinking waters from unprotected sources, and environmental sanitation problems.

Hymenolepis nana prevalence in the study area was $4.2 \%$. The result was relatively similar to the studies from Sudan (2.8\%) [28], Bahir Dar, Ethiopia (4.7\%) [29], Delo-Mena District Ethiopia (5.3\%) [35], Homesha District, Northwest 
Ethiopia (1.26\%) [33], Jawi town, Ethiopia (4.2\%) [19], Birbir town, Southern Ethiopia (1.7\%) [34], and Sasiga District, Southwest Ethiopia (5.7\%) [27]. However, it was lower than from Sudan (19\%) [17] and Gobgob, Northwest Ethiopia (14\%) [39]. The outcome was higher than the study from the Glomekeda district, Northern Ethiopia (0.7\%) [47]. These differences might be due to differences in animal contact especially with cats and dogs, eating raw vegetables, personal hygiene, the habit of playing with soil, the habit of sucking fingers, and eating raw meat.

The prevalence of E. vermicularis in the study area was $4.2 \%$. The outcome was similar to the studies from DeloMena district, Ethiopia (1.2\%) [35], Gobgob, Northwest Ethiopia (7\%) [39], Sudan (1.20\%) [17], Homesha district, Northwest Ethiopia (1.77\%) [33], and Adigrat town, Northern Ethiopia (3.56\%) [36]. However, it was higher than the prevalence from Iran (0.07\%) [18], Birbir town, Southern Ethiopia (0.6\%) [34], and Bahir Dar, Ethiopia (0.3\%) [29]. The outcome was lower than the study done in China (54.86\%) [59]. Drinking water from unprotected sources, sucking fingers, and learning materials, and eating raw vegetables might be reasons for E. vermicularis infection in the study area.

The prevalence of T. trichiura in the study area was $0.5 \%$ and it was the lowest among the nine species identified from the study area. The study was relatively similar to the studies done in Nigeria (3.57\%) [60], Delo-Mena district, Ethiopia (1.6\%) [35], Gobgob, Northwest Ethiopia (4.8\%) [39], Homesha district, Northwest Ethiopia (0.75\%) [33], Birbir town, Southern Ethiopia (5.7\%) [34], Adigrat town, Northern Ethiopia (3.24\%) [36], Bahir Dar, Ethiopia (2.8\%) [29], Gurage zone, Southcentral Ethiopia (5\%) [37], Bahir Dar Zuria (3\%) [57], and Glomekeda district, Northern Ethiopia (0.5\%) [47]. However, it was lower than other studies reported from Sasiga District, Southwest Ethiopia (7.6\%) [27], Nigeria (14.4\%) [56], Malaysia (53.8\%) [50], Southwestern China (25.2\%) [58], Gedeo Zone, Southern region of Ethiopia (75.2\%) [30], and East Arsi, Ethiopia (8.4\%) [40]. The variation in T. trichiura infection could be attributed to variations in feces disposal (use feces as fertilizer in agriculture), working on a farm, and contamination of food and water.

The predominant IPIs in the five primary schools were Entamoeba sp., hookworm, and S. mansoni. The prevalence of hookworm was $33 \%, 22 \%, 31 \%, 14.7 \%$, and $17.1 \%$ among students from Gibtsawit, Enbosa Maseria, Wagira, Mirafe Mariam, and Korata primary schools, respectively. Likewise, the respective prevalence of Entamoeba sp. among Gibtsawit, Enbosa Maseria, Wagira, Mirafe Mariam, and Korata primary school students was 30\%, 30\%, 25.9\%, 25.7\%, and $35.2 \%$. S. mansoni prevalence was $16.7 \%, 15.5 \%, 13.4 \%$, $6.4 \%$, and $6 \%$ among students from Gibtsawit, Wagira, Korata, Mirafe Mariam, and Enbosa Maseria primary schools, respectively. These differences might be due to differences in walking barefoot, eating raw vegetables, drinking and the use of water from unprotected sources, swimming in contaminated water bodies, participation in agricultural and irrigation activities, plying with soil, using feces as fertilizers, open field defecations, and handwashing habits.
The present study showed that family size was strongly associated with IPIs. The likelihood of being infected by IPIs among students belonging to a family size of above five was twofold compared to students belonging to a family size of less than or equal to five. The present finding agrees with other studies conducted in Ethiopia [29, 37, 38]. Increasing the family size may increase overcrowding that in turn may lead to contamination with each other. Besides, families with large sizes may face economic problems so that their members may face undernutrition, poor sanitation, poor medication, and poor personal hygiene.

Students who lack knowledge of IPIs were twofold more likely to be infected than students who knew IPIs. This finding agrees with the studies done in Malaysia [50, 61]. The possible reason might be that students who did not know may have practiced activities that expose them to IPIs. Similarly, students may not protect their personal and environmental hygiene, could play with the soil, and practice open field defecation.

Open field school waste disposal activities in the study area were strongly associated with the prevalence of IPIs. Students from schools that practiced open field waste disposal activities were sixteen times prone to IPIs compared to students from schools that did not openly dispose of wastes. The outcome agrees with the study from Ethiopia [33]. Open field waste disposal activity might be a source of infection since students may touch wastes while playing on the ground. In the study area, $28.8 \%$ of the schools practiced open field waste disposal activities.

The odds of having Entamoeba sp. infection among school children who used to drink river water were twofold higher than that of their counterparts. This was in line with the studies reported by Hailegebriel and Nasiri et al. [29, 52]. River waters might be contaminated by flooding and wastes from domestic animals. Likewise, students who ate soil were fourfold more likely to be infected by Entamoeba sp. than their counterparts. This might be because the soil could contain amoeba cysts. However, geophagia was not previously reported as a risk factor for Entamoeba sp. infection.

Mothers' handwashing by water alone and not using school toilets by students were risk factors associated with G. lamblia infection. The odds of having G. lamblia infection among students whose mothers did wash their hands with water alone were eightfold higher than students whose mothers used to wash their hands with soap and water. On the other hand, students from mothers who did not wash their hands at all were twofold more likely to be infected with G. lamblia than their counterparts. This might be due to the fact unwashed hands could have a cyst of G. lamblia. Washing hands only by water may not clean the microcyst of G. lamblia from their hands.

Students who did not use the school toilet were four times more likely to be infected by G. lamblia than students who used the school toilet. Open defecation in the school compound might result in infection by G. lamblia while students play in the school compound, or perform outreach activities.

Students who used to drink unprotected water were 1.378 times more likely to be infected by E. vermicularis than 
students who used to drink chlorinated and boiled water (protected water). Drinking water sources might be infected due to open field defecation, human and animal contact, and wind. The outcome disagreed with the studies reported in Thailand, China, and Marshall Islands [59, 62, 63].

Grade and the school toilet function were risk factors for Taenia sp. (Table 4). The odds of having Taenia sp. in grades 1-4 was twofold more likely to be infected than that of students in grades 5-8. Being free to play on the ground, eating unwashed vegetables and fruits, eating raw meat, drinking raw milk, and poor personal hygiene among children who were in grades 1-4 may be the possible reasons for their infection.

Not using the school toilet was associated with a Taenia sp. infection. Taenia sp. infection increased threefold in students who did not use school toilets compared to students who used school toilets. Children who used open defecation in their school compounds may have disseminated Taenia sp. infection to their healthy friends. All schools in the study area did not have handwashing facilities after toilet use. The ova and proglottids of Taenia sp. might pass from student to student during playing.

Eating raw meat was the major associated risk factor for A. lumbricoides infection in the study area. Children who ate raw meat were fifteen times more likely to be infected by A. lumbricoides than children who did not eat raw meat. The study outcome disagrees with the findings from the Gurage zone, Southcentral Ethiopia [37]. Personal hygiene of slaughters and contaminated butchering areas might be the reason for the difference. In Ethiopia, especially in the countryside, there are no specified abattoirs. People perform slaughters on open grounds so that the chance of contamination with soil and soil-transmitted infections may be high.

4.1. Limitations. The study was limited to only wet-mount and formol-ether concentration techniques. Not using KatoKatz, PCR, and Ziehl-Neelsen techniques may underestimate the prevalence of IPIs in the area.

\section{Conclusions}

The prevalence of IPIs is high in the study locality. No clear difference in the prevalence of IPIs exists across the five schools. Family size greater than five, open field school waste disposal activities, and lack of knowledge of IPIs among children are independently associated with the overall prevalence of IPIs. We recommend community-based health education, mass treatment, and school-based deworming without interruption.

\section{Data Availability}

The data supporting the conclusions of this article are within the article and the supplementary file.

\section{Conflicts of Interest}

The authors declare that they have no conflicts of interest.

\section{Authors' Contributions}

Dires Tegen and Destaw Damtie contributed to conceptualization, data curation, methodology, and writing of the original draft and final draft. Dires Tegen contributed to investigation, software, and supervision.

\section{Acknowledgments}

The authors acknowledge the support from Hamusit and Korata Health Centers for permeating the laboratory facilities for stool sample testing.

\section{Supplementary Materials}

S1_File. Pdf. Ethical clearance paper. S2_File. Pdf. Questionnaire (English and Amharic versions). (Supplementary Materials)

\section{References}

[1] J. A. Rozendaal, Vector Control: Methods for Use by Individuals and Communities, World Health Organization, Geneva, Switzerland, 1997.

[2] I. Abdullah, H. Tak, F. Ahmad, N. Gul, S. Nabi, and T. Sofi, "Predominance of gastrointestinal protozoan parasites in children: a brief review," Journal of Health Education Research and Development, vol. 4, no. 4, 2016.

[3] World Health Organization, Working to Overcome the Global Impact of Neglected Tropical Diseases: First WHO Report on Neglected Tropical Diseases, World Health Organization, Geneva, Switzerland, 2010.

[4] P. J. Hotez and A. Kamath, "Neglected tropical diseases in sub-Saharan Africa: review of their prevalence, distribution, and disease burden," PLoS Neglected Tropical Diseases, vol. 3, no. 8, Article ID e412, 2009.

[5] World Health Organization, Soil-transmitted Helminth Infections, World Health Organization, Geneva, Switzerland, 2016.

[6] Federal Democratic Republic of Ethiopia Ministry of Health, Second Edition of National Neglected Tropical Diseases Master Plan, Federal Democratic Republic of Ethiopia Ministry of Health, Addis Ababa, Ethiopia, 2016.

[7] TSEDU-Ethiopia, National ODF Campaign 2024: Campaign Framework Document (2019/20-2023/24), Federal Democratic Republic Of Ethiopia, Addis Ababa, Ethiopia, 2019.

[8] C. T. Leshargie, A. Alebel, A. Negesse et al., "Household latrine utilization and its association with educational status of household heads in Ethiopia: a systematic review and metaanalysis," BMC Public Health, vol. 18, no. 1, p. 901, 2018.

[9] T. Workneh, A. Esmael, and M. Ayichiluhm, "Prevalence of intestinal parasitic infections and associated factors among Debre Elias primary schools children, East gojjam zone, Amhara region, north west Ethiopia," Journal of Bacteriology \& Parasitology, vol. 5, no. 1, 2014.

[10] E. Tefera, J. Mohammed, and H. Mitiku, "Intestinal helminthic infections among elementary students of Babile town, eastern Ethiopia," The Pan African medical journal, vol. 20, no. 1, p. 50, 2015.

[11] F. A. Al-Braiken, "Is intestinal parasitic infection still a public health concern among Saudi children," Saudi Medical Journal, vol. 29, no. 11, pp. 1630-1635, 2008. 
[12] G. Mwanjali, C. Kihamia, D. V. C. Kakoko et al., "Prevalence and risk factors associated with human Taenia solium infections in Mbozi District, Mbeya Region, Tanzania," PLoS Neglected Tropical Diseases, vol. 7, no. 3, Article ID e2102, 2013.

[13] M. L. Wani, A. A. Rather, F. Q. Parray et al., "Wandering ascaris coming out through the abdominal wall," International Journal of Preventive Medicine, vol. 4, no. 6, pp. 720-2, 2013.

[14] World Health Organization, Helminth Control In School Age Children: A Guide For Managers Of Control Programs, vol. 1, WHO, Geneva, Switzerland, 2011, http://whqlibdoc.who.int/ publications/2011/9789241548267_eng.pdf.

[15] P. Ayeh-Kumi, S. Quarcoo, G. Kwakye-Nuako, J. Kretchy, A. Osafo-Kantanka, and S. Mortu, "Prevalence of intestinal parasitic infections among food vendors in Accra, Ghana," Journal of Tropical Medicine \& Parasitology, vol. 32, no. 1, pp. 1-8, 2009.

[16] J. V. Mbuh, H. N. Ntonifor, and J. T. Ojong, "The incidence, intensity and host morbidity of human parasitic protozoan infections in gastrointestinal disorder outpatients in Buea Sub Division, Cameroon," The Journal of Infection in Developing Countries, vol. 4, no. 1, pp. 038-043, 2010.

[17] H. S. Siddig, I. A. Mohammed, M. N. Mohammed, and A. M. Bashir, "Prevalence of intestinal parasites among selected group of primary school children in Alhag Yousif Area, Khartoum, Sudan," International Journal of Medical Research \& Health Sciences, vol. 6, no. 8, pp. 125-131, 2017.

[18] F. Bahrami, A. Haghighi, G. Zamini, M. B. Khadem-Erfan, and E. Azargashb, "Prevalence and associated risk factors of intestinal parasitic infections in Kurdistan province, northwest Iran," Cogent Medicine, vol. 5, no. 1, Article ID 1503777, 2018.

[19] B. Sitotaw, H. Mekuriaw, and D. Damtie, "Prevalence of intestinal parasitic infections and associated risk factors among Jawi primary school children, Jawi town, north-west Ethiopia," BMC Infectious Diseases, vol. 19, no. 1, p. 341, 2019.

[20] Central Statistics Authority, Population And Housing Census Of Ethiopia, Central Statistics Authority, Addis Ababa, Ethiopia, 2007.

[21] Ethiopian Public Health Instutute, Improving Health Extension: Program in Ethiopia: An Evidence-Based. Policy Brief, Ethiopian Public Health Instutute, Addis Ababa, Ethiopia, 2014.

[22] L. Naing, T. Winn, and B. Rusli, "Practical issues in calculating the sample size for prevalence studies," Archives of Orofacial Sciences, vol. 1, pp. 9-14, 2006.

[23] K. Williams, D. Thomson, I. Seto et al., "Standard 6: age groups for pediatric trials," Pediatrics, vol. 129, pp. S153-S160, 2012.

[24] World Health Organization, Basic Laboratory Methods in Medical Parasitology (Archived), WHO, Geneva, Switzerland, 1991.

[25] D. W. Hosmer Jr, S. Lemeshow, and R. X. Sturdivant, Applied Logistic Regression, John Wiley \& Sons, New York, NY, USA, 2013.

[26] World Health Organization, WHO Model Prescribing Information: Drugs Used in Parasitic Diseases, WHO, Geneva, Switzerland, 2nd edition, 1995.

[27] B. Sitotaw and W. Shiferaw, "Prevalence of intestinal parasitic infections and associated risk factors among the first-cycle primary schoolchildren in Sasiga district, southwest Ethiopia," Journal of parasitology research, vol. 2020, Article ID 8681247, 13 pages, 2020.
[28] M. Suliman, A. Magboul, H. Mohammed, A. Tamomh, and H. Bakhit, "Prevalence of intestinal parasitic infections and associated risk factors among school children in white nile state, Sudan," Journal of Infectious Diseases \& Diagnosis, vol. 4 , no. 125 , p. 2, 2019.

[29] T. Hailegebriel, "Prevalence of intestinal parasitic infections and associated risk factors among students at Dona Berber primary school, Bahir Dar, Ethiopia," BMC Infectious Diseases, vol. 17, no. 1, p. 362, 2017.

[30] H. Hailu, B. Lindtjørn, and B. Lindtjorn, "Intestinal parasites among rural schoolchildren in southern Ethiopia: a crosssectional multilevel and zero-inflated regression model," Maternal And Child Health In South Ethiopia, vol. 19, 2019.

[31] L. Chelkeba, Z. Mekonnen, Y. Alemu, and D. Emana, "Epidemiology of intestinal parasitic infections in preschool and school-aged Ethiopian children: a systematic review and meta-analysis," BMC Public Health, vol. 20, no. 1, p. 117, 2020.

[32] M. Lewetegn, M. Getachew, T. Kebede, G. Tadesse, and T. Asfaw, "Prevalence of intestinal parasites among preschool children and maternal KAP on prevention and control in Senbete and Bete towns, North Shoa, Ethiopia," International Journal of Biomedical Materials Research, vol. 7, no. 1, pp. 1-7, 2019.

[33] G. Weidengus, "Current status of intestinal parasites and associated risk factors among schoolchildren of Homesha District in Northwest Ethiopia," International Journal of Current Research, vol. 9, no. 7, pp. 54369-54374, 2017.

[34] G. Alemu, A. Abossie, and Z. Yohannes, "Current status of intestinal parasitic infections and associated factors among primary school children in Birbir town, Southern Ethiopia," BMC Infectious Diseases, vol. 19, no. 1, p. 270, 2019.

[35] B. Tulu, S. Taye, Y. Zenebe, and E. Amsalu, "Intestinal parasitic infections and nutritional status among primary school children in delo-mena district, south eastern Ethiopia," Iranian Journal of Parasitology, vol. 11, no. 4, pp. 549-558, 2016.

[36] D. S. Maru, "Prevalence of intestinal parasitic infections and associated risk factors among school children in Adigrat town, northern Ethiopia," International Journal of Emerging Trends in Science and Technology, vol. 4, no. 1, pp. 4943-4948, 2015.

[37] B. Melesse, U. Kelebesa, and P. Beyene, "Prevalence and determinants of intestinal parasitic infections among primary schoolchildren in Gurage zone, South Central Ethiopia," EC Microbiol, vol. 8, no. 2, pp. 59-70, 2017.

[38] A. A. Fentahun, A. Asrat, A. Bitew, and S. Mulat, "Intestinal parasitic infections and associated factors among mentally disabled and non-disabled primary school students, Bahir Dar, Amhara regional state, Ethiopia, 2018: a comparative cross-sectional study," BMC Infectious Diseases, vol. 19, no. 1, p. 549, 2019.

[39] M. Alemu, A. Anley, and K. Tedla, "Magnitude of intestinal parasitosis and associated factors in rural school children, Northwest Ethiopia," Ethiopian journal of health sciences, vol. 29, no. 1, pp. 923-928, 2019.

[40] M. Tadesse, B. Dobo, and M. Birmeka, "Prevalence and associated risk factors of intestinal parasitic infections among school children in Bamo no. 2 primary school, Adele town, East Arsi, Ethiopia," Sub-Saharan African Journal of Medicine, vol. 6, no. 2, p. 77, 2019.

[41] S. A. Vilwanathan, A. L. J. Yashwanth, B. Kandasamy, and T. Vijayalakshmi, "Prevalence of intestinal parasitic infections and predisposing factors among children in field practice area of tertiary care centre in South India," Age, vol. 5, pp. 5-10, 2017. 
[42] K. Ismail, "Prevalence of intestinal parasitic infection among school children in Taif," Insights Biomed, vol. 3, no. 2, p. 10, 2018.

[43] E. Achi, O. Njoku, A. Nnachi et al., "Prevalence of intestinal parasitic infections among under five children in Abakaliki local government area of Ebonyi state," Ejpmr, vol. 4, no. 4, pp. 218-222, 2017.

[44] E. Butera, A. Mukabutera, E. Nsereko et al., "Prevalence and risk factors of intestinal parasites among children under two years of age in a rural area of Rutsiro district, Rwanda-a crosssectional study," The Pan African Medical Journal, vol. 32, 2019.

[45] K. Sylla, R. Kouly Tine, D. Sow, and S. Lelo, "Epidemiological profile of intestinal parasitic infection among preschool and school children living in a rural community in Senegal: a cross sectional survey," Journal of Bacteriology \& Parasitology, vol. 9 , no. 343 , p. 2, 2018.

[46] A. Jejaw, E. Zemene, Y. Alemu, and Z. Mengistie, "High prevalence of Schistosoma mansoni and other intestinal parasites among elementary school children in Southwest Ethiopia: a cross-sectional study," BMC Public Health, vol. 15, no. 1, pp. 600-607, 2015.

[47] A. Dessie, T. G. Gebrehiwot, B. Kiros, S. D. Wami, and D. H. Chercos, "Intestinal parasitic infections and determinant factors among school-age children in Ethiopia: a crosssectional study," BMC Research Notes, vol. 12, no. 1, p. 777, 2019.

[48] C. W. Liao, K. C. Chiu, I. C. Chiang et al., "Prevalence and risk factors for intestinal parasitic infection in schoolchildren in Battambang, Cambodia," The American Journal of Tropical Medicine and Hygiene, vol. 96, no. 3, pp. 583-588, 2017.

[49] L. Galgamuwa, D. Iddawela, and S. Dharmaratne, "Intestinal protozoa infections, associated risk factors and clinical features among children in a low-income tea plantation community in Sri Lanka," International Journal of Community Medicine and Public Health, vol. 3, no. 9, pp. 2452-2458, 2016.

[50] O. E. Tunbosun, L. Rampal, H. A. Rahman, and R. A. Majid, "Prevalence and predictors of intestinal worm infections among semai aboriginal tribe school children in tapah, Malaysia," Malaysian Journal of Medicine and Health Sciences, vol. 13, no. 1, pp. 27-34, 2017.

[51] D. R. Venkatajothi, "Incidence of intestinal protozoa infections among school going children," International Journal of Current Research in Medical Sciences, vol. 3, no. 4, pp. 54-58, 2017.

[52] V. Nasiri, K. Esmailnia, G. Karim, M. Nasir, and O. Akhavan, "Intestinal parasitic infections among inhabitants of Karaj City, Tehran province, Iran in 2006-2008," Korean Journal of Parasitology, vol. 47, no. 3, p. 265, 2009.

[53] M. Babakhani, R. Safari, F. Rajati, S. Salimi, and A. Omidian Doost, "Prevalence and risk factors associated with intestinal parasitic infections among school children in Gashky, West of Iran," International Journal of Pediatrics, vol. 5, no. 7, pp. 5263-5273, 2017.

[54] M. Osman, D. El Safadi, A. Cian et al., "Prevalence and risk factors for intestinal protozoan infections with Cryptosporidium, Giardia, Blastocystis and Dientamoeba among schoolchildren in Tripoli, Lebanon," PLoS Neglected Tropical Diseases, vol. 10, no. 3, Article ID e0004496, 2016.

[55] M. B. Shiferaw and A. D. Mengistu, "Helminthiasis: hookworm infection remains a public health problem in Dera district, South Gondar, Ethiopia," PLoS One, vol. 10, no. 12, Article ID e0144588, 2015.
[56] A. Ugbomeh, D. Goodhead, A. Green, and J. Onwuteaka, "Prevalence of human intestinal nematode parasites in three rural communities of the Niger delta, Nigeria," Asian Journal of Biology, vol. 6, no. 3, pp. 1-10, 2018.

[57] A. Amor, E. Rodriguez, J. M. Saugar et al., "High prevalence of Strongyloides stercoralis in school-aged children in a rural highland of north-western Ethiopia: the role of intensive diagnostic work-up," Parasites \& Vectors, vol. 9, no. 1, pp. 617-618, 2016.

[58] D. Yang, Y. Yang, Y. Wang et al., "Prevalence and risk factors of Ascaris lumbricoides, Trichuris trichiura and Cryptosporidium infections in elementary school children in southwestern China: a school-based cross-sectional study," International Journal of Environmental Research and Public Health, vol. 15, no. 9, Article ID 1809, 2018.

[59] H. M. Li, C. H. Zhou, Z. S. Li et al., "Risk factors for Enterobius vermicularis infection in children in Gaozhou, Guangdong, China," Infectious diseases of poverty, vol. 4, no. 1, p. 28, 2015.

[60] L. Muazu, Y. Abdullahi, and Z. Umar, "Prevalence of human intestinal parasitic nematode among out-patients attending wudil general hospital, kano state, Nigeria," Annals of Tropical Medicine and Public Health, vol. 6, no. 3, pp. 1-5, 2017.

[61] L. Quihui-Cota, G. G. Morales-Figueroa, A. Javalera-Duarte, J. A. Ponce-Martínez, E. Valbuena-Gregorio, and M. A. López-Mata, "Prevalence and associated risk factors for Giardia and Cryptosporidium infections among children of northwest Mexico: a cross-sectional study," BMC Public Health, vol. 17, no. 1, p. 852, 2017.

[62] C. Nithikathkul, B. Changsap, S. Wannapinyosheep, C. Poister, and P. Boontan, "The prevalence of Enterobius vermicularis among primary school students in Samut Prakan Province, Thailand," Southeast Asian Journal of Tropical Medicine and Public Health, vol. 32, pp. 133-137, 2001.

[63] C.-K. Fan, T.-W. Chuang, Y.-C. Huang et al., "Enterobius vermicularis infection: prevalence and risk factors among preschool children in kindergarten in the capital area, Republic of the Marshall Islands," BMC Infectious Diseases, vol. 19, no. 1, p. 536, 2019. 